\title{
CLASSIFICATION AND PREDICTION OF RF COUPLING INSIDE A-320 AND A-319 AIRPLANES USING FEED FORWARD NEURAL NETWORKS
}

\author{
Madiha Jafri, Old Dominion University, Norfolk, Virginia \\ Jay Ely, NASA Langley Research Center, Hampton, Virginia \\ Dr. Linda Vahala, Old Dominion University, Norfolk, Virginia
}

\begin{abstract}
:
Neural Network Modeling is introduced in this paper to classify and predict Interference Path Loss measurements on Airbus 319 and 320 airplanes. Interference patterns inside the aircraft are classified and predicted based on the locations of the doors, windows, aircraft structures and the communication/navigation system-of-concern. Modeled results are compared with measured data and a plan is proposed to enhance the modeling for better prediction of electromagnetic coupling problems inside aircraft.
\end{abstract}

\section{Introduction:}

Several modeling techniques have been proposed to address the concern about electromagnetic interference (EMI) caused by the use of Portable Electronic Devices (PEDs) onboard commercial airplanes. The EMI caused by these devices may endanger the safety of passengers. PEDs may act as transmitters, both intentional and unintentional, and their signals may be detected by various receivers on the aircraft. Researchers at NASA Langley Research Center, Eagles Wing Incorporated, and United Airlines have collected measurement data in an effort to understand the EMI pattern on aircraft. Previous publications include graphical and statistical models of Interference Path Loss (IPL) on several United A319 and A320 airplanes. IPL is the measurement of the radiated field coupling between passenger cabin locations and aircraft communication and navigation receivers, via their antennas. The measurement is required for assessing the threat of PEDs to aircraft radios, and is very dependant upon airplane size, the interfering transmitter's position within the aircraft, and the location of the particular antenna for the aircraft system of concern.

\section{Testing Methodology:}

Before attempting to understand the analysis of IPL data, it is necessary to review how the data was measured. IPL, as addressed herein, is particularly focused upon in-band, on-channel type EMI to aircraft radios, via their antennas. This does not include EMI to aircraft radios outside their radio frequency (RF) passband, and does not include radiated field (or conducted) coupling to wiring and equipment apertures.

\section{IPL Data}

IPL data was taken by radiating a low powered continuous wave (CW) test signal, frequencysynchronized to the spectrum analyzer sweep and fed to the test transmitting antenna via a doubleshielded RF cable. The spectrum analyzer, laptop computer controller, signal generators, RF amplifiers and preamplifiers were located inside the aircraft. The spectrum analyzer input cable was connected to the aircraft radio receiver rack cable in the avionics equipment bay.

To perform an IPL measurement, the team measured the RF power loss between the calibrated signal source and a spectrum analyzer, via the entire length of test cables plus the aircraft cable, plus the free space loss between the reference antenna and the aircraft antenna. To obtain a calibrated IPL measurement, test cable losses were measured separately by connecting the two ends of the test cables to the input and output of the spectrum analyzer, and subtracting this loss, in $\mathrm{dB}$, from the raw measurement. Individual IPL measurements were obtained by moving the test antenna from one window to the next, throughout the airplane. A complete description of the measurement process may be found in [1]. 


\section{Test Systems and Locations:}

IPL measurements were taken along all windows of A320 and A319 airplanes. From an electromagnetic coupling point-of-view, the A319 and A320 airplanes are structurally different, with different numbers of windows and exits, as well as with aircraft antennas installed at different locations. Figure 1 includes a side profile for the A319 and A320. As described previously, IPL measurements were taken with respect to each of the systems pointed to in Figure 1. Measurements were taken at each window location of the aircraft, on both port and starboard side, resulting in 32 measurements for the A319 and 40 measurements for the A320. Table 1 includes a list of the systems tested along with their operating frequencies.
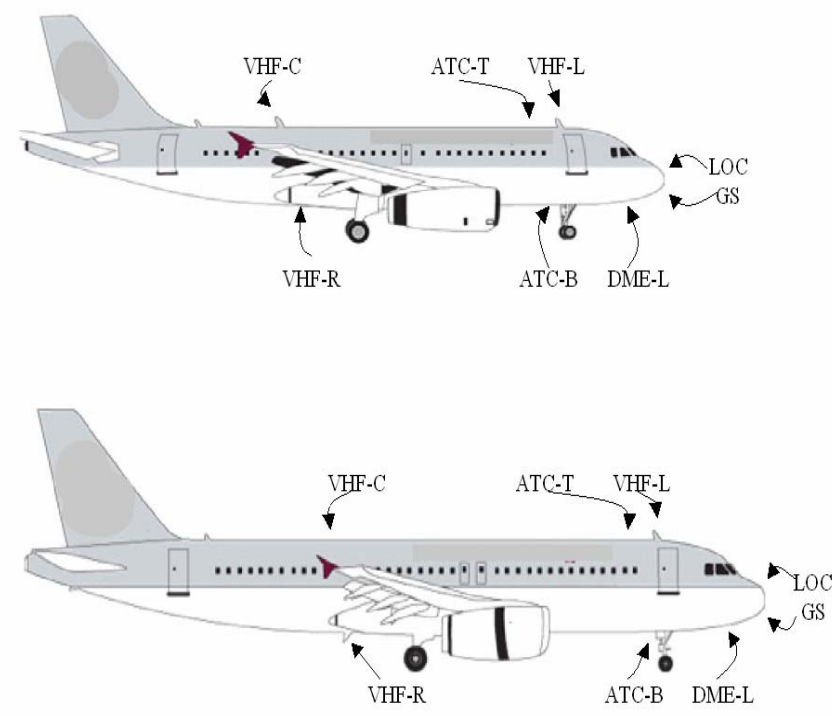

Figure 1: Aircraft systems tested on A-319 (top) and A-320 (bottom)

Table 1: Frequency Spectrums of Systems tested

\begin{tabular}{|l|l|}
\hline Aircraft Systems & Frequency Spectrum \\
\hline LOC & $108-118 \mathrm{MHz}$ \\
\hline GS & $325-340 \mathrm{MHz}$ \\
\hline DME & $960-1215 \mathrm{MHz}$ \\
\hline ATC & $1090 \mathrm{MHz}$ \\
\hline \multicolumn{1}{|c|}{ VHF } & $118-138 \mathrm{MHz}$ \\
\hline
\end{tabular}

IPL data was taken on both port and starboard sides of the aircraft. Due to the symmetry of the aircraft, the two measurement sets from port and starboard were considered two trials for each system, instead of two independent measurements. Eight systems were tested on the A319 (with two trials each for port and starboard, resulting in 16 datasets for the A319). Seven systems (with two trials) were tested on the A320, resulting in 14 datasets. Therefore, a total of 30 datasets were available for training and testing of neural networks as explained in the next sections. For each of the 30 datasets, data was taken in both vertical and horizontal polarizations.

\section{Neural Network Modeling}

Modeling techniques, such as ray tracing and fuzzy logic, have been proposed to study the interference patterns inside commercial aircraft due to PEDs; however, we suggest that none of the other modeling techniques can provide the useful results with as little computational effort as Neural networks. Neural networks not only have the capability to learn various interference patterns according to the locations of doors, windows and aircraft antenna location, but they also learn interference patterns from one aircraft to the other. This dynamic capability can significantly improve modeling accuracy for other aircraft, which may eventually eliminate the need to take time consuming and tedious IPL measurements of other aircraft.

\section{Introduction to Neural Networks}

Feed-forward neural networks have been widely used for various tasks, such as pattern recognition, function approximation, dynamical modeling, data mining, time-series forecasting and more. Many solutions in different fields have been obtained that were otherwise impossible through other modeling techniques, such as Markov models and complex computational models. Artificial neural networks (ANNs) are computational systems whose architecture and operation are inspired from the knowledge about biological neural cells (neurons) in the brain. ANNs can be described either as mathematical and computational models for non-linear function approximation, data classification, clustering and non-parametric regression or as simulations of the behavior of collections of model biological neurons. Neural modeling has shown incredible capability for emulation, analysis, prediction, and association. ANNs can be used in a variety of powerful ways: 
to learn and reproduce rules or operations from given examples; to analyze and generalize from sample facts and make predictions from these; or to memorize characteristics and features of given data and to match or make associations from new data to the old data.

The backpropagation algorithm is the most important algorithm for the supervised training of multi-layer feed-forward ANNs. It derives its name from the fact that error signals are propagated backward through the network on a layer-by-layer basis. The backpropagation algorithm is based on the selection of a suitable error function or cost function, whose values are determined by the actual and desired outputs of the network and which is also dependant on the network parameters such as the weights and the thresholds.

The layer which intakes the input values is known as the input layer; similarly, the last layer is known as the output layer. The layers in between the input and output layers are known as hidden layers and consist of several arbitrary number of nodes. Figure 2 is a good illustration of a feedforward neural network structure in which three layers are shown. The dashed arrows going upward show the application of the backpropagation algorithm used to update the two weight matrices labeled at the two interconnections of the three layers.

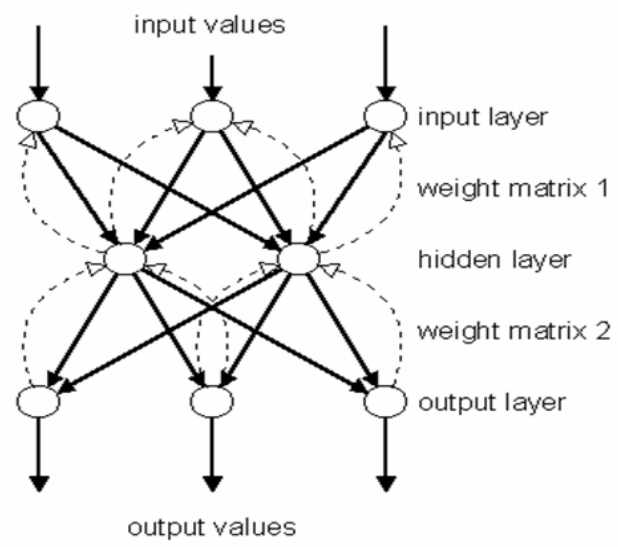

Figure 2: Feed Forward Neural Network

The main goal of backpropagation is to train weights such that they minimize the squared error described as follows:

$$
E=\frac{1}{2} \sum_{n=1}^{N} \sum_{k=1}^{K}\left[y_{k}^{n}-f_{k}\left(x^{n}\right)\right]^{2}
$$

where $\mathrm{n}$ is the number of training samples, $\mathrm{k}$ is the number of output units; the values being subtracted are $\mathrm{y}_{\mathrm{k}}$, the target output with $\mathrm{f}_{\mathrm{k}}(\mathrm{x})$, which is the output produced by the neural network undergoing training or testing. In backpropagation, the weights of the network are updated starting with the hidden to output weights, followed by the input to hidden weights, with respect to the sum of square error mentioned above and through a series of weight update rules, called the delta rules.

\section{ANN Structure used for IPL data}

A three-layer ANN model was created with one input layer, one hidden layer and one output layer.

\section{Input Layer:}

For each of the 30 datasets, 13 different characteristics were fed into the Neural networks as inputs. These characteristics are shown in Table 2:

Table 2: Input characteristics for ANN

\begin{tabular}{|c|c|c|}
\hline Features & A319 & A320 \\
\hline Aircraft Length (cm) & 3383 & 3750 \\
\hline Port vs. Starboard & 1 or 0 & 1 or 0 \\
\hline Number of Windows & 32 & 40 \\
\hline Exit 1 location & 767 & 780 \\
\hline Exit 2 location & 2810 & 3050 \\
\hline Emergency Exit 1 loc. & 1604 & 1002 \\
\hline Emergency Exit 2 loc. & 1604 & 1709 \\
\hline Aircraft system loc. (x) & $200 \rightarrow 3383$ & $200 \rightarrow 3750$ \\
\hline Aircraft system loc. (y) & $-206.8 \rightarrow+206.8$ & $-206.8 \rightarrow+206.8$ \\
\hline Aircraft system loc. (z) & $-15 \rightarrow+15$ & $-15 \rightarrow+15$ \\
\hline Op. freq. (start, MHz) & $108 \rightarrow 1565$ & $108 \rightarrow 1565$ \\
\hline Op. freq. (stop, MHz) & $108 \rightarrow 1585$ & $108 \rightarrow 1585$ \\
\hline System’s dominant pol. & $\mathrm{H}(0)$ or $\mathrm{V}(1)$ & $\mathrm{H}(0)$ or $\mathrm{V}(1)$ \\
\hline
\end{tabular}

In the table, the various lengths and aircraft system and exit locations were determined through detailed schematic drawings, provided by the courtesy of United Airlines. These measurements, represented in centimeters, refer to the station (STA) numbers, provided in the schematics.

The objective of this work was also to create a single neural network to learn about two structurally different aircraft. For example, the 
A319 only has one emergency exit (at STA 1604), while the A320 has two over wing emergency exits (at STA1002 and STA1709). These inconsistencies were taken into consideration by reserving two input nodes for emergency exits and duplicating the emergency exit location for the A319. Therefore, nodes 6 and 7 in Table 2 are STA1002 and STA1709 respectively for the A320; however, both nodes are STA1604 for the A319.

\section{Output Layer:}

As per the objective of this research, the output nodes consisted of the IPL measurements (in $\mathrm{dB}$ ) at each window location. Therefore, 32 output nodes are required for the A319 and 40 output nodes are required for the A320. However, in order to create a single neural network for both structurally different aircraft, same number of output nodes was necessary. To avoid data loss, A319 data was duplicated at 8 locations (every $4^{\text {th }}$ window) to result in a total of 40 windows.

\section{Final ANN Structure:}

Ideally, two 13-input, 40-output neural networks should have been used for this simulation (one for horizontal and vertical polarization, respectively). The top structure in Figure 3 shows a 13-input, 40-output ANN. The number of hidden nodes is dependant on the number of inputs and outputs. Although no particular formula is available to calculate the number of hidden nodes, too many or too few hidden nodes can cause the ANN to under-learn or over-learn during training, thus leading to inaccurate results during testing. Assuming that 20 hidden nodes were needed for efficient classification, this would result in 13x20 inputhidden connections and 20x40 hidden-output connections, resulting in a total of 1060 nodeto-node connections. The ANN model for 1060 nodes required more processing power than was readily available for the time constraints of this analysis. Due to computer resource limitations, as well as for a more efficient and faster design, four 20-output, neural networks were instead created: two for vertical polarization in the front and rear half of the airplane and two more for horizontal polarization in the front and rear airplane halves. Each of the four feed forward, back propagation network were created with 13 inputs, 15 hidden units in hidden layer, and 20 units in the output layer. This structure had $13 \times 15$ input-hidden connections and 15x20 hidden-output connections, resulting in a total of 495 connections.

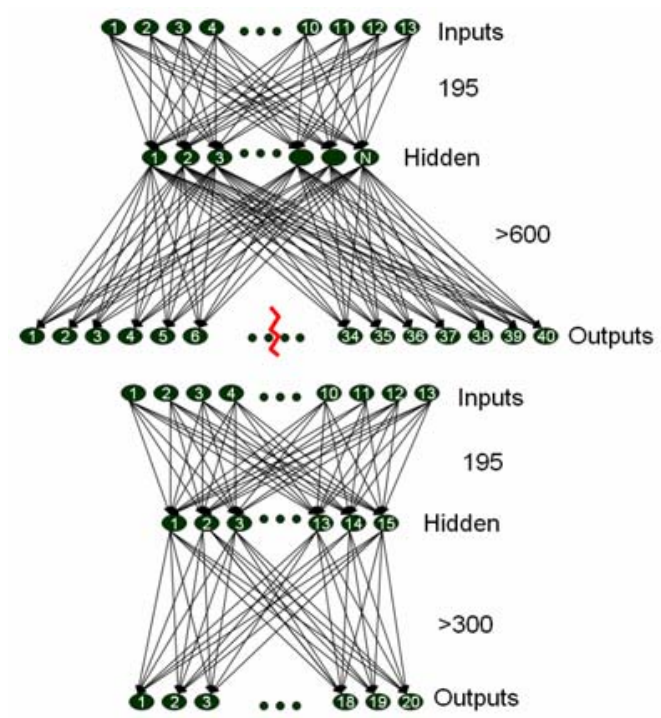

Figure 3: Original ANN structure (top) vs. Final Structure (bottom)

\section{ANN Modeling Results}

Using the ANN structure defined in Section 2, IPL data was used to train the ANN and then testing was performed to estimate the accuracy of the trained ANN.

\section{Training and Testing Data Division:}

Training data inputs and outputs are used to set the weights of the ANN properly, with the goal of minimizing the error rate. Once the weights are set (using the training set's outputs and back propagation algorithm), testing data input is passed through the ANN structure, and the computed outputs are compared with actual 
testing data's outputs to determine the accuracy of the ANN prediction. Table 3 shows the systems tested for each aircraft type.

Table 3: Available data from each aircraft type

\begin{tabular}{|c|c|}
\hline A319 & A320 \\
\hline ATC-B & ATC-B \\
\hline ATC-T & ATC-T \\
\hline DME-L & DME-L \\
\hline GS-L & GS-L \\
\hline LOC-L & LOC-L \\
\hline VHF-C & VHF-C \\
\hline VHF-L & VHF-L \\
\hline VHF-R & \\
\hline
\end{tabular}

Fifteen ANN simulations were performed as per the availability of data in Table 3 . In each simulation, one system was reserved as test system, while all the other fourteen systems were used to train the ANN. For example, for the first simulation, A319's ATC-B was reserved to test the $A N N$, while all other systems (A319's ATC-T, DME-L, GS-L, LOCL, VHF-C, VHF-L, VHF-R, A320's ATC-B, ATC-T, DME-L, GS-L, LOC-L, VHF-C and VHF-L) were used to train the ANN.

\section{ANN Results}

Figures 4 to 18 are the results from each of the fifteen simulations. System tested by the ANN is specified in the caption of each figure. The light (green) lines represent the training data (from the other fourteen systems in the training set), while the solid (red) represents the test data (unknown to the ANN). The dashed line (red) represents the ANN output based on the 13 inputs of the test dataset. Please refer to Table 4 for a comparative analysis of ANN results.

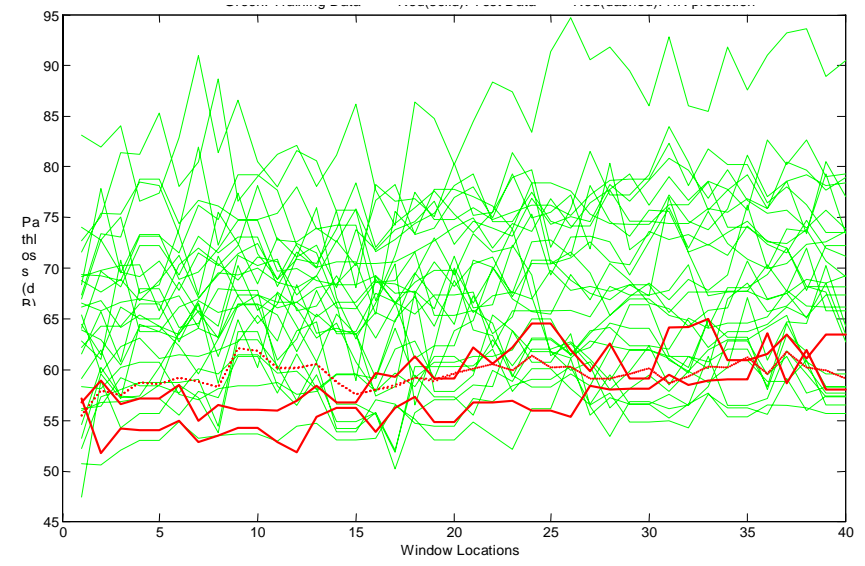

Figure 4: A319- ATC-B (Vertical Polarization)

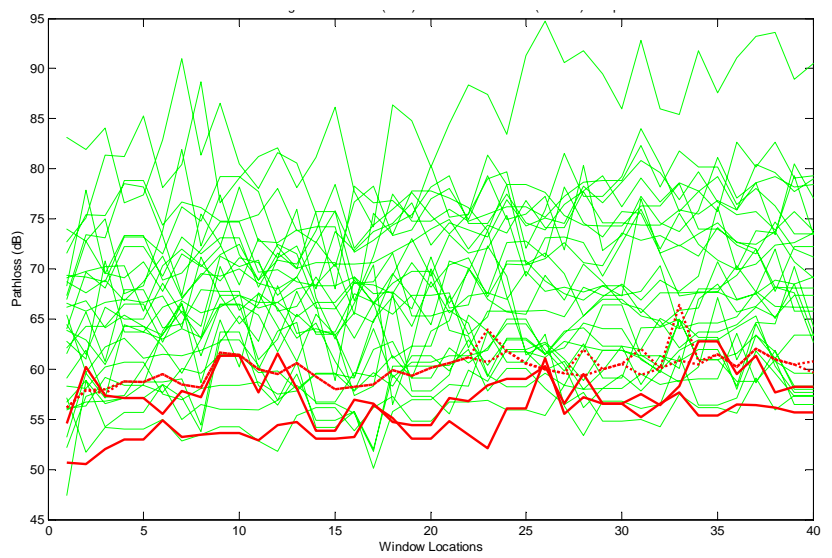

Figure 5: A319- ATC-T (Vertical Polarization)

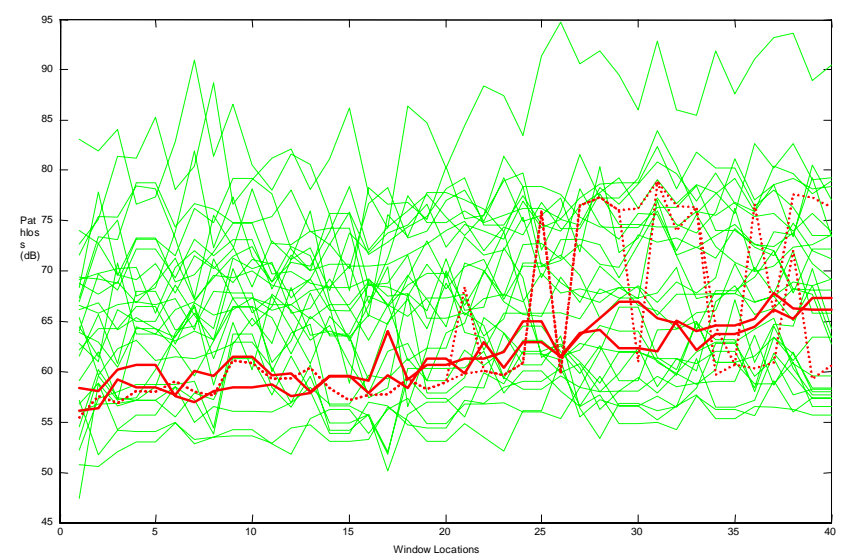

Figure 6: A319- DME-L (Vertical Polarization) 


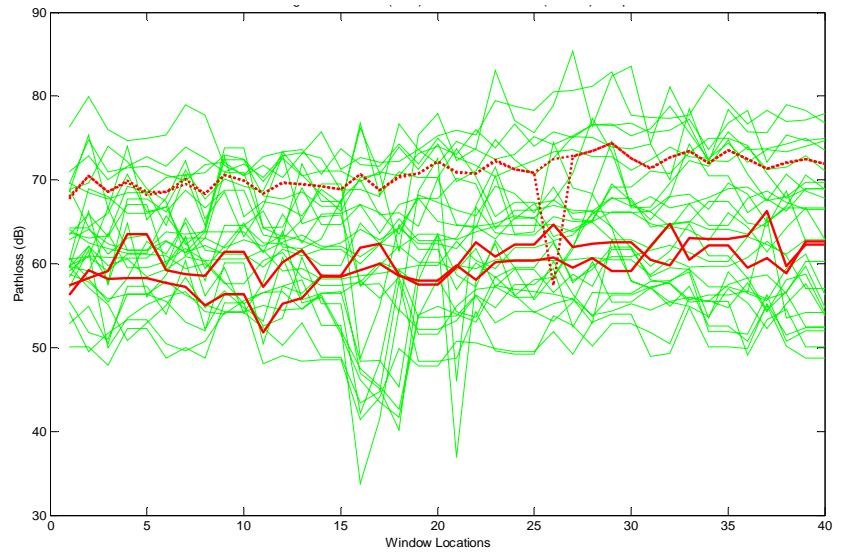

Figure 7: A319- GS-L (Horizontal P.)

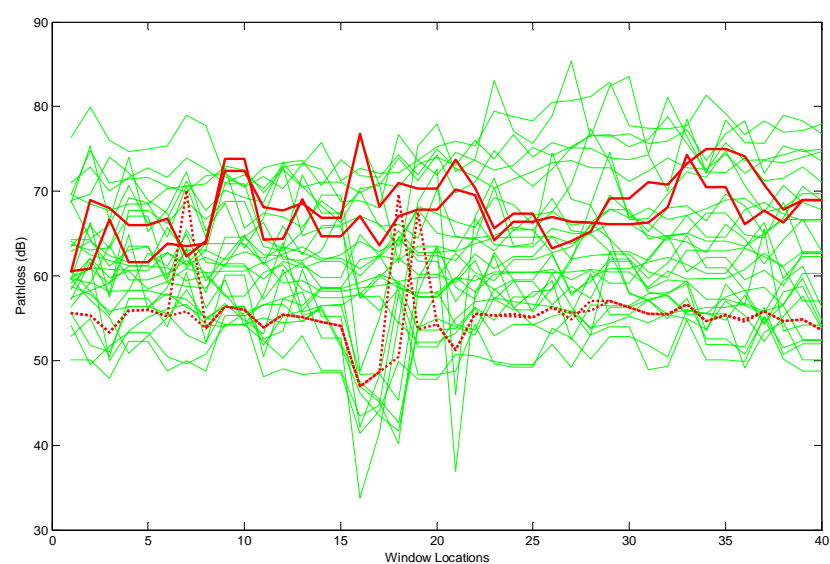

Figure 8: A319- LOC-L (Horizontal P.)

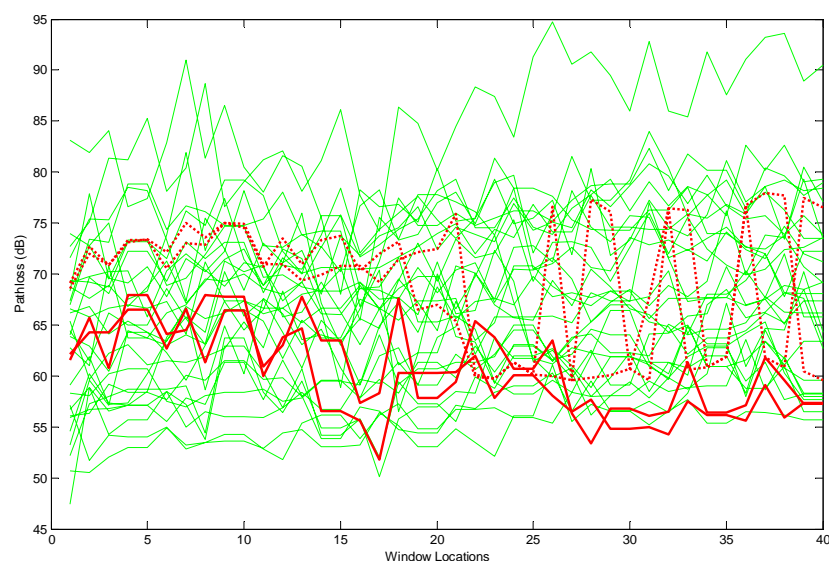

Figure 9: A319-VHF-C (Vertical P.)

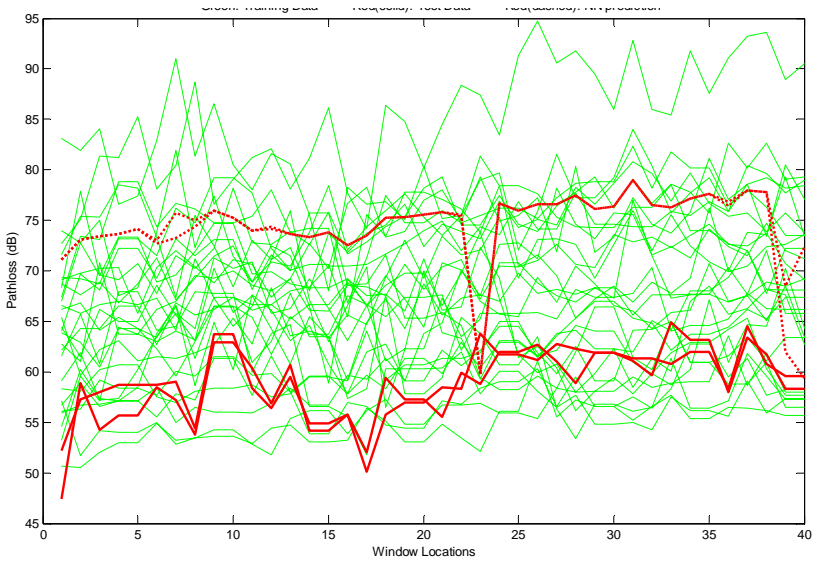

Figure 10: A319- VHF-L (Vertical P.)

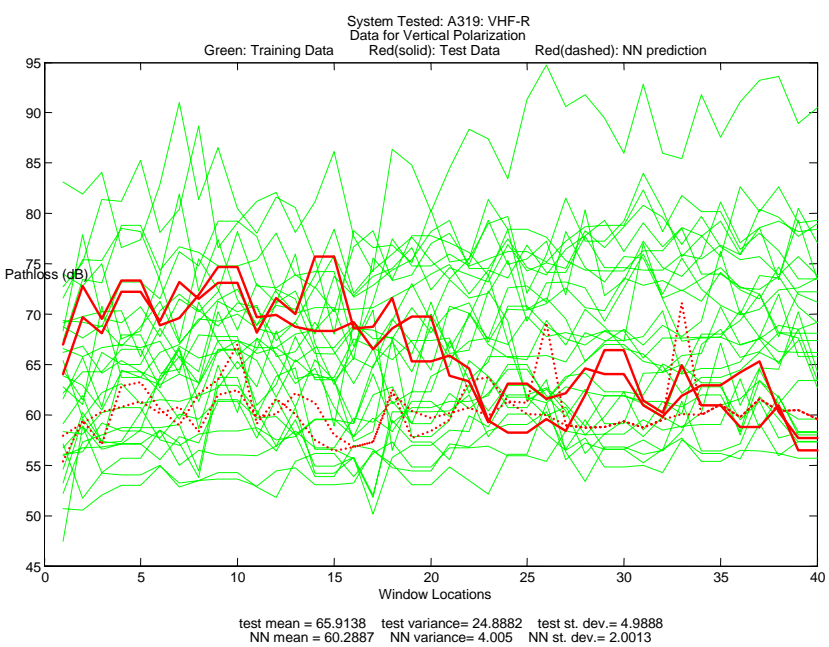

Figure 11: A319- VHF-R (Vertical P.)

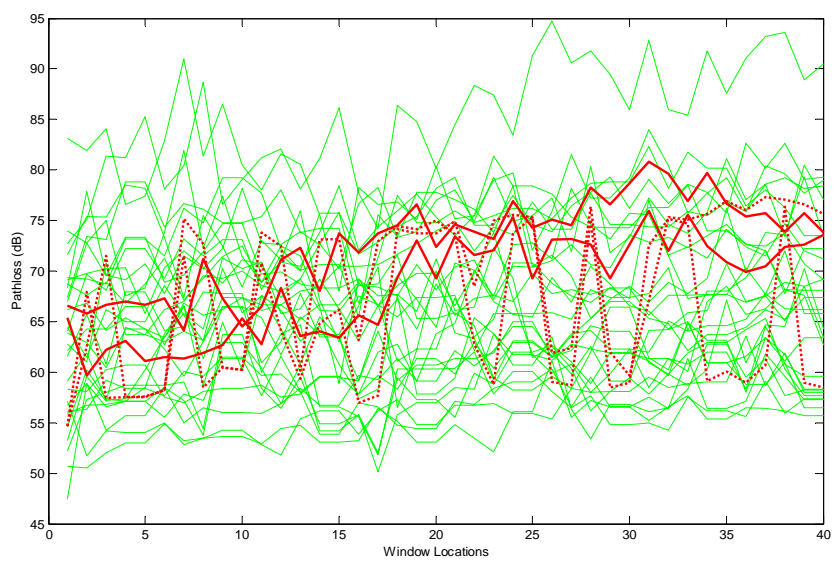

Figure 12: A320- ATC-B (Vertical P.) 


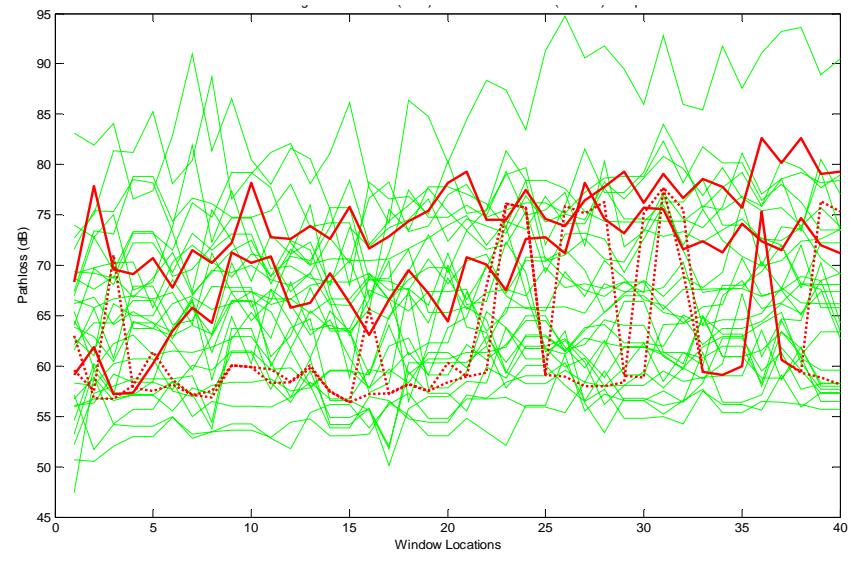

Figure 13: A320- ATC-T (Vertical P.)

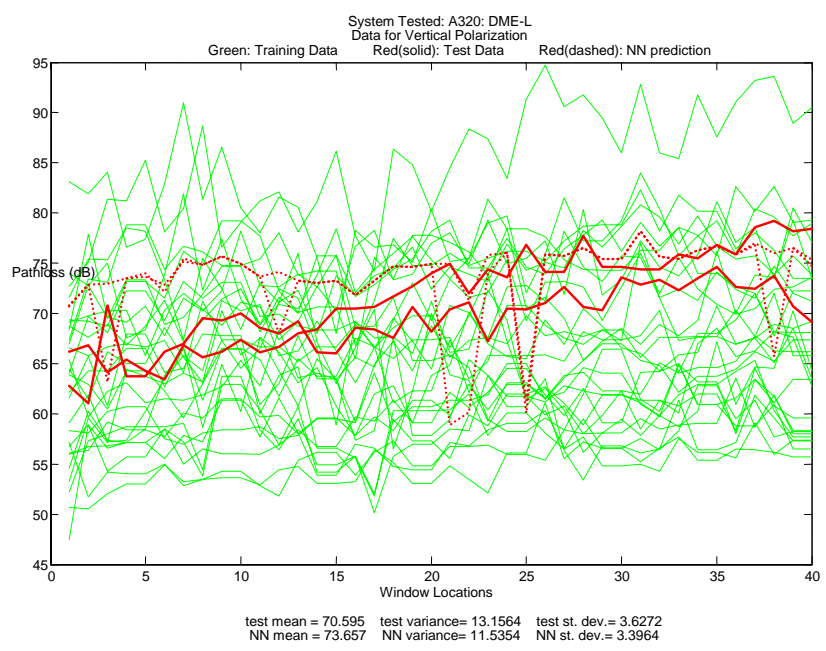

Figure 14: A320- DME-L (Vertical P.)

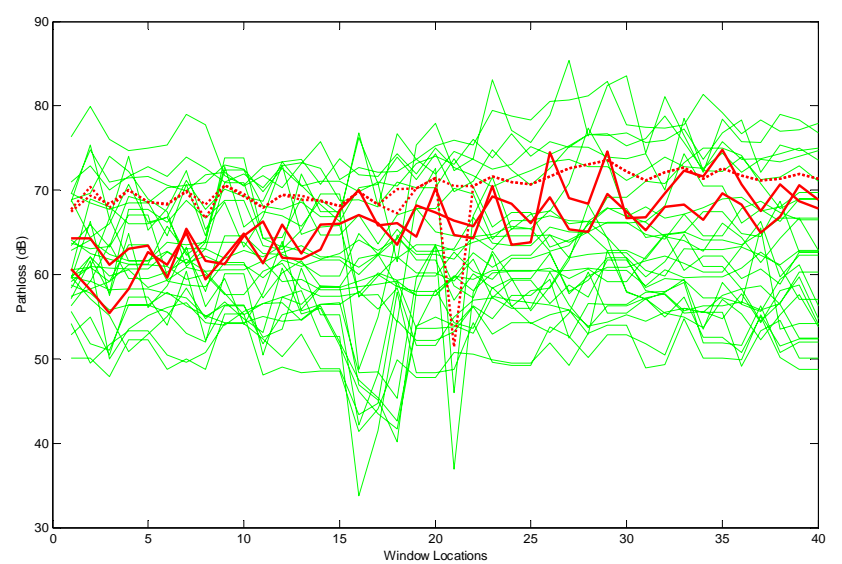

Figure 15: A320-GS-L (Horizontal P.)

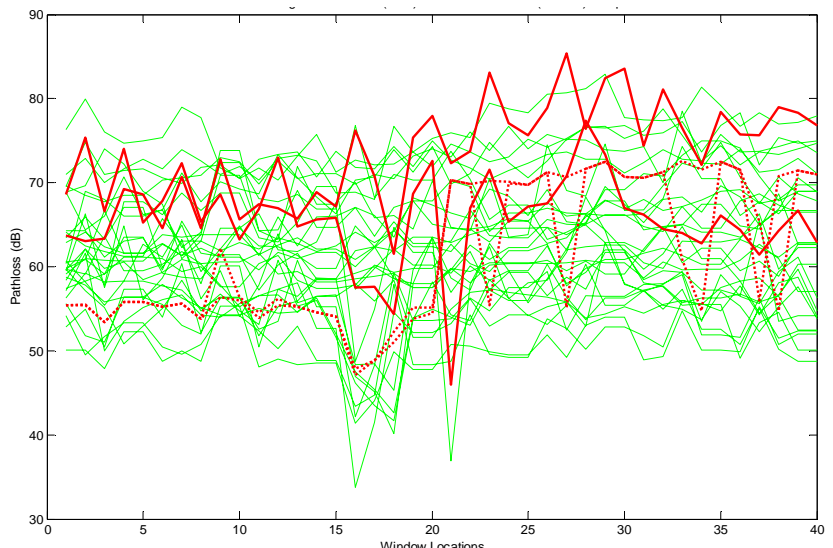

Figure 16: A320-LOC-L (Horizontal P.)

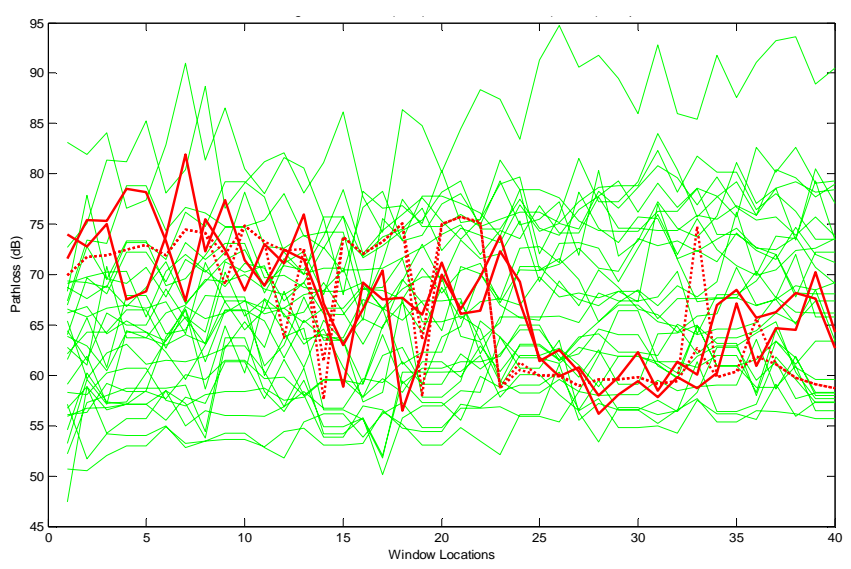

Figure 17: A320- VHF-C (Vertical P.)

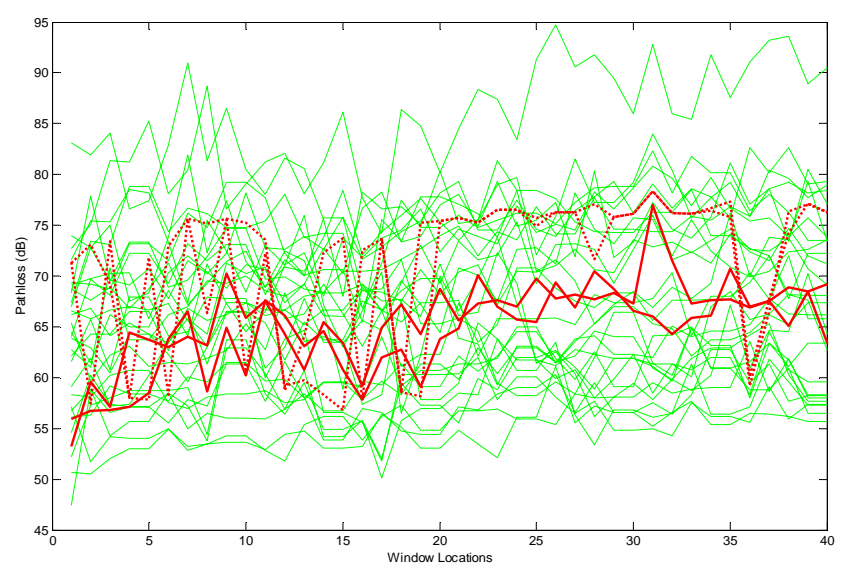

Figure 18: A320- VHF-L (Vertical P.) 
Table 4: Actual vs. ANN IPL comparison

\begin{tabular}{|c|c|c|c|c|c|}
\hline & System & $\begin{array}{l}\text { Actual } \\
\text { Mean }\end{array}$ & $\begin{array}{l}\text { ANN } \\
\text { Mean }\end{array}$ & $\begin{array}{l}\text { Actual } \\
\text { Std. }\end{array}$ & $\begin{array}{c}\text { ANN } \\
\text { Std. }\end{array}$ \\
\hline \multirow{8}{*}{$\frac{9}{m}$} & ATC-B & 58.1 & 59.5 & 2.5 & 1.3 \\
\hline & ATC-T & 56.3 & 59.9 & 1.7 & 1.5 \\
\hline & DME-L & 61.6 & $\begin{array}{l}63.6 \\
\end{array}$ & 2.9 & 7.2 \\
\hline & GS-L & 60.0 & 70.7 & 2.0 & 1.9 \\
\hline & LOC-L & 67.8 & 55.3 & 3.1 & 2.6 \\
\hline & VHF-C & 60.4 & 68.9 & 3.8 & 4.9 \\
\hline & VHF-L & 59.1 & 74.4 & 3.3 & 3.6 \\
\hline & VHF-R & 65.9 & 60.3 & 4.9 & 2.0 \\
\hline \multirow{7}{*}{ ڤิ } & ATC-B & 70.6 & 67.2 & 4.5 & 6.2 \\
\hline & ATC-T & 72.0 & 62.4 & 4.1 & 5.9 \\
\hline & DME-L & 70.6 & 73.6 & 3.6 & 3.4 \\
\hline & GS-L & 66.0 & 70.1 & 3.4 & 2.3 \\
\hline & LOC-L & 69.6 & 61.5 & 4.6 & 7.9 \\
\hline & VHF-C & 67.2 & 66.7 & 5.5 & 6.7 \\
\hline & VHF-L & 65.1 & 71.1 & 3.7 & 6.2 \\
\hline
\end{tabular}

Table 4 shows the means and standard deviations for actual IPL data versus ANN simulated IPL data for all fifteen simulations. ATC-B and DME-L tested exceptionally well with very close means and standard deviations for both A319 and A320 simulations. The maximum difference of $15.5 \mathrm{~dB}$ occurred in prediction of A319's VHF-L. The A319's actual VHF-L mean is $59.1 \mathrm{~dB}$, while A320's VHF-L has an actual mean of $65.1 \mathrm{~dB}$. When the A319's VHF-L was being tested, the ANN used A320's VHF-L as a reference, along with correlation to other systems to predict the IPL pattern for A319's VHF-L. Therefore, although there is error between actual and predicted data, the higher IPL prediction for A319's VHF-L shows that the ANN indeed learned from other systems and predicted accordingly. ANN prediction errors will continue to decline as more training data is incorporated. Past comparisons of measurement data from B737 and B747 airplanes showed variations of 3 to $6 \mathrm{~dB}$ between similar airplanes, so 3 to $6 \mathrm{~dB}$ variations between ANN predictions and measured IPL data should be considered optimal. [6,7]
It is also interesting to note that A319's DME$\mathrm{L}$ has a mean of $61.6 \mathrm{~dB}$, while A320's DME-L has a mean of $70.6 \mathrm{~dB}$. The ANN performs very well in predicting DME-L patterns for both aircraft, showing that the ANN is in fact also dynamically learning from other systems, instead of simply copying A320 DME-L results for A319 prediction and vice versa. The same dynamic learning applies to ATC-B for both aircraft.

Also, VHF-R was only tested on the A319 (and not on the A320); therefore, when testing the A319's VHF-R, the ANN did not have reference to another aircraft's VHF-R patterns. Learning from all non-VHF-R systems, the ANN was able to predict the A319's VHF-R pattern very efficiently with only a $5 \mathrm{~dB}$ mean difference.

\section{Conclusion}

Modeling of IPL inside commercial aircraft has been a concern for many years. Although several modeling techniques have been proposed, the techniques have been too complex or inflexible for practical application to various structurally different aircraft. Neural network modeling, based on artificial intelligence, is an excellent selection for this application, due to its ability to learn and predict various IPL patterns from one aircraft to another for various system antennas installed in different aircraft locations.

The analysis presented in this paper shows that measurement data from two structurally different aircraft can be integrated together for effective ANN learning. With vigorous simulations, ANNs were trained to predict IPL patterns inside commercial aircraft, depending on the locations of exits, locations of antennas, the length and structure of aircraft as well as the location, operating frequency and polarization of the aircraft system of concern. Incorporation of other aircraft, such as B757 and B737 should further enhance the modeling. Most importantly, the measurement and ANN simulation results are statistically comparable. Statistical comparisons are necessary for developing risk analyses for EMI caused by PEDs being used on board airplanes.

\section{Acknowlegment}

The data evaluated in this paper were obtained from a joint partnership between United Airlines, 
Eagles Wings Inc. and NASA's Aviation Safety Program. The authors specifically thank Mr. Gerald L. Fuller for his previous analysis of the data upon which this work builds.

\section{References}

[1]. Jafri, Madiha, J. Ely, L. Vahala. "Graphical and Statistical Analysis of Airplane Passenger Cabin RF Coupling Paths to Avionics.” $22^{\text {nd }}$ Digital Avionics Systems Conference, Indianapolis, IN, October 12 - 16, 2003. http://techreports.larc.nasa.gov/ltrs/PDF/2003/ mtg/NASA-2003-22dasc-mj.pdf

[2]. Fuller, Gerald. "B737-200 Path Loss Tests: Victorville, California.” Mariposa, CA, March 2002, Task 1 report under NASA Contract L16099

[3]. Horton, Kent; Huffman, Mitch; Eppic, Brian; White, Harrison, "757 Path Loss Measurements”, NASA/CR-2005-213762, 20050601; June 2005. http://hdl.handle.net/2060/20050186545

[4]. RTCA/DO-233, "Portable Electronic Devices Carried on Board Aircraft”, August 20, 1996.

[5]. Jafri, Madiha, J. Ely, L. Vahala. ”Classification and Prediction of Interference Pathloss Measurements inside B757 Using Feed Forward Neural Networks.” $12^{\text {th }}$ Biennial IEEE Conference on Electromagnetic Field Computation, Miami, FL, April 30 - May 3, 2006

[6]. Fuller, Gerald, "B747-400 Path Loss Tests, Victorville, California, April 2002”, NASA PO L-16099 Task 2, May 2002.

[7]. Fuller, Gerald, "B737-200 Path Loss Tests, Victorville, California, May 2002”, NASA PO L-16099 Task 3, June 2002. 